\title{
What are the implications for policy makers? A systematic review of the cost-effectiveness of screening and brief interventions for alcohol misuse in primary care
}

\author{
Colin Angus ${ }^{1}{ }^{*}$, Nicholas Latimer ${ }^{1}$, Louise Preston ${ }^{1}$, Jessica Li ${ }^{1}$ and Robin Purshouse ${ }^{2}$ \\ 1 School of Health and Related Research (ScHARR), University of Sheffield, Sheffield, UK \\ ${ }^{2}$ Department of Automatic Control and Systems Engineering, University of Sheffield, Sheffield, UK
}

Edited by:

Antoni Gual, Hospital Clínic de Barcelona, Spain

\section{Reviewed by:}

Antoni Gual, Hospital Clínic de Barcelona, Spain

Robert F. Leeman, Yale School of

Medicine, USA

\section{*Correspondence:}

Colin Angus, School of Health and Related Research (ScHARR),

University of Sheffield, Regents

Court, 30 Regent Street, Sheffield S1

4DA, UK

e-mail: c.r.angus@sheffield.ac.uk

\begin{abstract}
Introduction: The efficacy of screening and brief interventions (SBIs) for excessive alcohol use in primary care is well established; however, evidence on their cost-effectiveness is limited. A small number of previous reviews have concluded that SBI programs are likely to be cost-effective but these results are equivocal and important questions around the cost-effectiveness implications of key policy decisions such as staffing choices for delivery of SBIs and the intervention duration remain unanswered.
\end{abstract}

Methods: Studies reporting both the costs and a measure of health outcomes of programs combining SBIs in primary care were identified by searching MEDLINE, EMBASE, Econlit, the Cochrane Library Database (including NHS EED), CINAHL, PsycINFO, Assia and the Social Science Citation Index, and Science Citation Index via Web of Knowledge. Included studies have been stratified both by delivery staff and intervention duration and assessed for quality using the Drummond checklist for economic evaluations.

Results: The search yielded a total of 23 papers reporting the results of 22 distinct studies. There was significant heterogeneity in methods and outcome measures between studies; however, almost all studies reported SBI programs to be cost-effective. There was no clear evidence that either the duration of the intervention or the delivery staff used had a substantial impact on this result.

Conclusion: This review provides strong evidence that SBI programs in primary care are a cost-effective option for tackling alcohol misuse.

Keywords: alcohol drinking, screening and brief intervention, primary care, systematic review, policy making, resource allocation, brief alcohol intervention, brief intervention

\section{INTRODUCTION}

The misuse of alcohol is a substantial concern for public health policy makers across the world, with over $5 \%$ of the global burden of disease and injury estimated as being alcohol-attributable (1). In addition to these deleterious effects on health and the associated economic costs, excessive consumption of alcohol is also associated with a range of social harms such as increased crime, public nuisance, and reduced workplace productivity, which impact not just on the drinker, but on society as a whole (2).

Primary care provides an avenue through which a large proportion of the population can be reached by interventions aimed at reducing alcohol misuse and the related consequences. In particular, excessive drinkers attend primary care with greater frequency than moderate drinkers (3) and may therefore be more easily targeted through this channel. Programs of Screening and Brief Interventions (SBIs), in which patients are screened opportunistically for alcohol misuse and those screening positively are offered a brief session of advice can harness these properties to achieve broad coverage of the population at risk (4).

There is a substantial body of existing research into the effectiveness of SBI programs in primary care, with a recent review of reviews identifying 24 previous systematic reviews (5). The consistent finding of these studies is that SBIs are effective at reducing excessive alcohol consumption and this weight of evidence has led to the inclusion of SBIs in a range of international policy recommendations including the World Health Organisation's global strategy for tackling harmful alcohol use (6). However, in spite of these calls for the implementation of such policies, evidence on the cost-effectiveness of SBI programs is less equivocal. This is a key question for the policy makers and healthcare budget planners being urged to instigate or fund these programs and there have been few attempts to draw together the existing literature in order to inform their decisions.

There have been three major previous reviews of the costeffectiveness evidence on SBIs in primary care (7-9). While all three conclude that they are cost-effective, none examine the impact that implementation decisions such as the staff used to deliver the SBI, or the duration of the intervention itself, have on overall program cost-effectiveness. These issues are critical as the use of general practitioners (GPs) to deliver SBIs is usually a substantially more expensive option than nursing staff and a lack of available time is the single greatest perceived barrier 
to early intervention in alcohol problems in primary care (10). In addition, these existing reviews either predate several important studies or have a narrow scope which misses a number of key papers. This study updates and expands the 2008 review by Latimer et al. (8) in order to provide a systematic overview of the existing cost-effectiveness evidence for SBIs in primary care, together with an examination of the differential impact of alternative implementation options.

\section{METHODS}

The original search was undertaken in May 2008 (8) and refreshed on four subsequent occasions, with the latest update undertaken in April 2014. Searches were conducted on the following electronic databases:

Medline in Process and Other Non-Indexed Citations and Medline 1950-present via OVID SP

EMBASE via OVID SP

Science Citation Index via Web of Knowledge

Social Science Citation Index via Web of Knowledge

Cochrane Library Databases via Wiley

Assia via CSA

PsycINFO via OVID SP

Econlit via OVID SP

The original search undertaken in 2008 adopted an iterative emergent approach. Rather than developing an a priori search strategy, smaller individual searches were undertaken in order to develop understanding of the research area. The information specialist (Louise Preston) and lead reviewer (Nicholas Latimer) worked together to develop further iterations of the search strategy based on the findings of earlier searches. As a result, for this update, the use of a predetermined search strategy was possible as search terms had been tested and validated as part of the original searches. The search strategy is presented in Figure 1.

The title and abstracts of all retrieved studies were screened by one reviewer (Colin Angus) against a set of pre-defined inclusion and exclusion criteria. These criteria, listed in Supplementary Material, were piloted with a second reviewer (Jessica Li) on an initial subsample of 10 studies and subsequently refined, following discussions between both reviewers, to ensure clarity in their interpretation. Any study reporting the costs and health or other economic benefits of SBI programs in primary care were considered for inclusion. Studies were excluded which were not published in English, which examined multi-behavior interventions (e.g., combined drink and drugs education programs), which included components occurring outside of primary care, or which evaluated interventions comprising more than four patient contacts (on the grounds that these no longer constitute "brief" interventions). Studies examining SBI implementation strategies only (e.g., GP education programs to increase delivery rates of SBIs to patients) were excluded unless they presented a separate economic evaluation of the SBI delivery itself. Similarly, studies that examined only screening tools (e.g., AUDIT or CAGE) were excluded unless they also included a BI component.

Data from all included studies were extracted by one reviewer (Colin Angus) using a standard template (see Supplementary

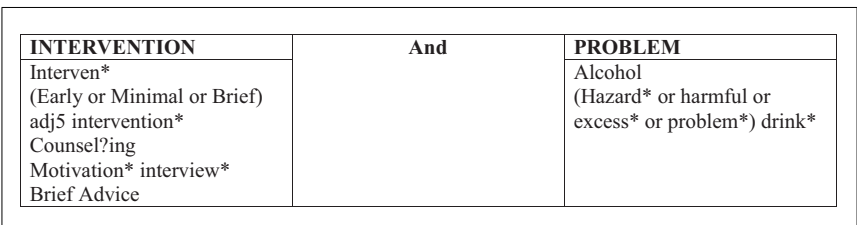

FIGURE 1 | Search strategy utilized in the review

Material) adapted from that used by Latimer et al. (8). Studies were assessed for methodological quality using the Drummond checklist for economic evaluations (11) as recommended for use in Cochrane reviews (12). Five of the included studies were randomly selected and additionally assessed for quality by a second reviewer (Jessica Li) to ensure consistency (agreement was $100 \%$ between both reviewers).

\section{RESULTS}

Twenty-three papers reporting the results of 22 distinct studies that met the criteria for inclusion in the review were identified. These fall into two major categories: economic evaluations alongside clinical trials (EEACTs) (13-21) and stand-alone modeling evaluations (4, 7, 22-33). Table 1 summarizes these studies, while excluded studies are reported in Supplementary Material. A glossary of relevant health economic terms is included in Supplementary Material.

These 23 studies examine the cost-effectiveness of SBIs in almost exclusively high-income countries (Chisholm et al. being the only exception (30)), with the majority of studies covering the USA ( 8 studies), UK ( 5 studies), or Australia (3 studies). There was considerable variation in the quality of the studies, with 7 rated as being of low quality, 10 of moderate quality, and 5 of high quality, although there are signs of an improving trend over time with more recent papers scoring more highly. The main issues encountered were an inadequate description of the intervention itself, poor reporting of the sources of cost data used in the studies, and insufficient sensitivity analysis.

Of the nine studies reporting evaluations alongside clinical trials, two compared different levels of brief intervention $(13,14)$, both concluding that a longer "stepped care" intervention was the most cost-effective option. Another six studies compared brief interventions with usual care $(15,17-21)$. The trials that these studies are associated with ran for between 6 and 48 months, while the full effect of changes in drinking behavior on health outcomes can take many years to develop (34). It is therefore perhaps unsurprising that these studies found few statistically significant results and do not allow any firm conclusions to be drawn around the cost-effectiveness of SBI programs.

All except one of the 14 modeling studies compared SBI provision to an alternative do-nothing scenario in which no SBIs are delivered. The other study (24) examined the cost-effectiveness of increasing the current uptake rate. Among these studies, the most common health outcome measures were QALYs $(4,23,25$, $26,28,31,33)$, with two studies using DALYs $(22,30)$ and two using life years gained $(7,32)$. Almost all these studies found SBIs to be either cost-saving and health improving (i.e., they dominate a do-nothing scenario) or to have very low costs relative to health 
Table 1 | Characteristics of included studies.

\begin{tabular}{|c|c|c|c|c|c|c|c|c|c|}
\hline Study & Country & $\begin{array}{l}\text { Study } \\
\text { type }\end{array}$ & Comparators & Costs included & $\begin{array}{l}\text { Health } \\
\text { outcomes } \\
\text { included }\end{array}$ & Results & Quality & $\begin{array}{l}\text { Duration of } \\
\text { intervention }\end{array}$ & $\begin{array}{l}\text { BI delivery } \\
\text { staff }\end{array}$ \\
\hline $\begin{array}{l}\text { Angus } \\
\text { et al. (28) }\end{array}$ & Italy & CUA & $\begin{array}{l}\text { (1) Do-nothing scenario ( } 2 \text { ) } \\
\text { Screening with AUDIT-C followed } \\
\text { by } 10 \text { min brief intervention }\end{array}$ & $\begin{array}{l}\text { Intervention costs and } \\
\text { health and social care } \\
\text { resource use over } \\
30 \text { years following start } \\
\text { of program }\end{array}$ & $\begin{array}{l}\text { QALYs gained } \\
\text { over } 30 \text { years } \\
\text { follow-up }\end{array}$ & $\begin{array}{l}\text { SBI delivered at next GP } \\
\text { registration has an ICER of } € 550 \\
\text { per QALY vs. do-nothing. SBI at } \\
\text { next GP consultation has an ICER } \\
\text { of } € 590 \text { per QALY vs. do-nothing. }\end{array}$ & ++ & $10 \mathrm{~min}$ & GP \\
\hline $\begin{array}{l}\text { Angus } \\
\text { et al. (33) }\end{array}$ & $\begin{array}{l}\text { Netherlands } \\
\text { and Poland }\end{array}$ & CUA & $\begin{array}{l}\text { (1) Do-nothing scenario } \\
\text { (2) Screening with AUDIT-C } \\
\text { followed by } 10 \text { min brief } \\
\text { intervention }\end{array}$ & $\begin{array}{l}\text { Intervention costs and } \\
\text { health and social care } \\
\text { resource use over } \\
30 \text { years following start } \\
\text { of program }\end{array}$ & $\begin{array}{l}\text { QALYs gained } \\
\text { over } 30 \text { years } \\
\text { follow-up }\end{array}$ & $\begin{array}{l}\text { Netherlands: SBI delivered at next } \\
\text { GP registration has an ICER of } \\
€ 6340 \text { per QALY vs. do-nothing. } \\
\text { SBI at next GP consultation has an } \\
\text { ICER of } € 5748 \text { per QALY vs. } \\
\text { do-nothing. Poland: SBI delivered } \\
\text { at next GP registration has an } \\
\text { ICER of zł3696 per QALY vs. } \\
\text { do-nothing. SBI at next GP } \\
\text { consultation has an ICER of zł3269 } \\
\text { per QALY vs. do-nothing. }\end{array}$ & ++ & $10 \mathrm{~min}$ & GP \\
\hline $\begin{array}{l}\text { Babor } \\
\text { et al. (15) }\end{array}$ & USA & $\begin{array}{l}\text { EEACT/ } \\
\text { CEA }\end{array}$ & $\begin{array}{l}\text { Screening with AUDIT followed by } \\
\text { either: (1) Treatment as usual } \\
\text { (2) } 3-5 \text { min brief intervention }\end{array}$ & Intervention costs & $\begin{array}{l}\text { SF-12 score and } \\
\text { mean alcohol } \\
\text { consumption at } \\
12 \text { months } \\
\text { follow-up }\end{array}$ & $\begin{array}{l}\text { Small but significant reduction in } \\
\text { consumption for BI group vs. } \\
\text { treatment as usual. No significant } \\
\text { difference in SF-12 scores. No } \\
\text { significant differences in either } \\
\text { outcome between GP- and } \\
\text { nurse-delivered intervention } \\
\text { groups }\end{array}$ & - & $3-5 \min$ & GP or nurse \\
\hline $\begin{array}{l}\text { Chisholm } \\
\text { et al. (30) }\end{array}$ & International & CUA & $\begin{array}{l}\text { (1) Do-nothing scenario } \\
\text { (2) Screening followed by brief } \\
\text { intervention involving four primary } \\
\text { care visits inside a year }\end{array}$ & Intervention costs & $\begin{array}{l}\text { DALYs averted } \\
\text { over a lifetime } \\
\text { horizon }\end{array}$ & $\begin{array}{l}\text { SBI varies from dominated by to } \\
\text { dominating a do-nothing scenario } \\
\text { depending on WHO region with } \\
9 / 12 \text { regions having an ICER of } \\
\leq 5000 \text { l\$ per } Q A L Y\end{array}$ & + & Not stated & GP \\
\hline $\begin{array}{l}\text { Cobiac } \\
\text { et al. (22) }\end{array}$ & Australia & CUA & $\begin{array}{l}\text { (1) Do-nothing scenario } \\
\text { (2) Screening followed by } \\
\text { counseling, supportive written } \\
\text { materials and follow-up } \\
\text { consultations with further advice } \\
\text { "if necessary" }\end{array}$ & $\begin{array}{l}\text { Intervention costs, } \\
\text { patient time/travel and } \\
\text { health and social care } \\
\text { resource use over } \\
\text { lifetime horizon }\end{array}$ & $\begin{array}{l}\text { DALYs averted } \\
\text { over a lifetime } \\
\text { horizon }\end{array}$ & $\begin{array}{l}\text { ICER of } \$ 6800 \text { per DALY averted } \\
\text { vs. do-nothing }\end{array}$ & - & Not stated & GP \\
\hline
\end{tabular}


Table 1 | Continued

\begin{tabular}{|c|c|c|c|c|c|c|c|c|c|}
\hline Study & Country & $\begin{array}{l}\text { Study } \\
\text { type }\end{array}$ & Comparators & Costs included & $\begin{array}{l}\text { Health } \\
\text { outcomes } \\
\text { included }\end{array}$ & Results & Quality & $\begin{array}{l}\text { Duration of } \\
\text { intervention }\end{array}$ & $\begin{array}{l}\text { BI delivery } \\
\text { staff }\end{array}$ \\
\hline $\begin{array}{l}\text { Dillie et al. } \\
\text { (16) }\end{array}$ & USA & $\begin{array}{l}\text { EEACT/ } \\
\text { Cost } \\
\text { mini- } \\
\text { mization } \\
\text { analysis }\end{array}$ & $\begin{array}{l}\text { Screening with self-reported } \\
\text { alcohol consumption followed by } \\
\text { either: } \\
\text { (1) } 2 \times 15 \text { min brief interventions } \\
\text { each followed up with a } 5 \text { min } \\
\text { telephone call } \\
\text { (2) Additional screened with \% } \\
\text { CDT followed by } 2 \times 15 \text { min brief } \\
\text { interventions each followed up } \\
\text { with a } 5 \text { min telephone call }\end{array}$ & $\begin{array}{l}\text { Intervention costs, } \\
\text { patient time/travel, } \\
\text { health and social care } \\
\text { resource use, motor } \\
\text { vehicle crashes and } \\
\text { legal/criminal costs over } \\
4 \text { years follow-up }\end{array}$ & N/A & $\begin{array}{l}\text { Addition of \% CDT screening } \\
\text { saves } \$ 212 \text { per patient screened }\end{array}$ & + & $40 \mathrm{~min}$ & $\begin{array}{l}\text { GP (nurse } \\
\text { delivers } \\
\text { follow-up } \\
\text { phone calls) }\end{array}$ \\
\hline $\begin{array}{l}\text { Drummond } \\
\text { et al. (14) }\end{array}$ & UK (Wales) & $\begin{array}{l}\text { EEACT/ } \\
\text { CUA }\end{array}$ & $\begin{array}{l}\text { Screening with AUDIT followed by } \\
\text { either: } \\
\text { (1) 5-min nurse-led "minimal } \\
\text { intervention" } \\
\text { (2) "Stepped care" - } 20 \text { min } \\
\text { behavioral change counseling } \\
\text { session followed up with referral } \\
\text { to motivational enhancement } \\
\text { therapy and/or specialist alcohol } \\
\text { services if indicated }\end{array}$ & $\begin{array}{l}\text { Intervention costs, } \\
\text { health and social care } \\
\text { resource use costs and } \\
\text { costs of crime at } \\
6 \text { months follow-up }\end{array}$ & $\begin{array}{l}\text { QALYs gained at } \\
6 \text { months follow } \\
\text { up }\end{array}$ & $\begin{array}{l}\text { Stepped care } 98 \% \text { likely to be } \\
\text { most cost-effective option at a } \\
\text { threshold of } £ 20,000-30,000 \text { per } \\
\text { OALY. No ICER presented }\end{array}$ & - & $\begin{array}{l}5 \text { min } \\
\text { (minimal } \\
\text { intervention) } \\
\text { or } 20+\text { min } \\
\text { (stepped } \\
\text { care) }\end{array}$ & $\begin{array}{l}\text { Practice } \\
\text { nurse }\end{array}$ \\
\hline $\begin{array}{l}\text { Fleming } \\
\text { et al. (17, } \\
18)\end{array}$ & USA & $\begin{array}{l}\text { EEACT/ } \\
\text { CBA }\end{array}$ & $\begin{array}{l}\text { Screening with 7-day timeline } \\
\text { follow back followed by either: } \\
\text { (1) Patient information leaflet } \\
\text { (2) } 2 \times 15 \text { min brief interventions } \\
\text { each followed up with a } 5 \text { min } \\
\text { telephone call }\end{array}$ & $\begin{array}{l}\text { Intervention costs, } \\
\text { patient time/travel, } \\
\text { health and social care } \\
\text { resource use, motor } \\
\text { vehicle crashes and } \\
\text { legal/criminal costs over } \\
\text { lifetime horizon }\end{array}$ & $\begin{array}{l}\text { Mean alcohol } \\
\text { consumption at } \\
\text { various points up } \\
\text { to } 4 \text { years } \\
\text { follow-up }\end{array}$ & $\begin{array}{l}\text { Significant reduction in } \\
\text { consumption observed in SBI } \\
\text { group ( } 32 \% \text { in men, } 43 \% \text { in } \\
\text { women). SBI estimated to save } \\
\$ 546 \text { per patient from healthcare } \\
\text { perspective and } \$ 7780 \text { from a } \\
\text { societal perspective vs. patient } \\
\text { information leaflet }\end{array}$ & + & $40 \mathrm{~min}$ & $\begin{array}{l}\text { GP (nurse } \\
\text { delivers } \\
\text { follow-up } \\
\text { phone calls) }\end{array}$ \\
\hline $\begin{array}{l}\text { Freeborn } \\
\text { et al. (19) }\end{array}$ & USA & $\begin{array}{l}\text { EEACT/ } \\
\text { Resource } \\
\text { utiliza- } \\
\text { tion } \\
\text { analysis }\end{array}$ & $\begin{array}{l}\text { Screening with AUDIT followed by } \\
\text { either: } \\
\text { (1) Treatment as usual } \\
\text { (2) Brief advice from GP then } \\
15 \text { min motivational session with } \\
\text { trained counselor }\end{array}$ & $\begin{array}{l}\text { Health and social care } \\
\text { resource use over } \\
2 \text { years follow-up }\end{array}$ & N/A & $\begin{array}{l}\text { No significant difference in health } \\
\text { and social care resource use } \\
\text { between } \mathrm{BI} \text { and care as usual } \\
\text { groups }\end{array}$ & - & $15+\min$ & $\begin{array}{l}\text { GP and } \\
\text { trained } \\
\text { counselor }\end{array}$ \\
\hline
\end{tabular}


Table 1 | Continued

Study Country

Study Comparators

Costs included

Health

Results

intervention

staff

included

Mean alcohol

SBI costs $£ 8-20$ per patient,

- $15 \mathrm{~min}$

GP

Freeman

(2) Screening with AUDIT followed

Intervention costs

consumption a

which equates to f18-47 per

by $15 \mathrm{~min}$ brief intervention

24 months

patient who reduces their

follow-up

drinking, with a mean reduction of

$24 \%$ among those who cut down

\begin{tabular}{|c|c|c|c|c|c|c|c|c|c|}
\hline $\begin{array}{l}\text { Kapoor } \\
\text { et al. (23) }\end{array}$ & USA & CUA & $\begin{array}{l}\text { (1) Do-nothing scenario } \\
\text { (2) Screening with AUDIT followed } \\
\text { by full clinical assessment of } \\
\text { unhealthy alcohol use and } \\
5-10 \text { min brief intervention } \\
\text { (3) Screening with AUDIT and \% } \\
\text { CDT followed by full clinical } \\
\text { assessment of unhealthy alcohol } \\
\text { use and 5-10 min brief } \\
\text { intervention }\end{array}$ & $\begin{array}{l}\text { Intervention costs, } \\
\text { health and social care } \\
\text { resource use over } \\
\text { lifetime horizon }\end{array}$ & $\begin{array}{l}\text { QALYs gained } \\
\text { over lifetime } \\
\text { horizon }\end{array}$ & $\begin{array}{l}\text { Both screening strategies } \\
\text { dominate vs. do-nothing. } \\
\text { Incremental cost of adding \% CDT } \\
\text { to screening is } \$ 15,500 \text { per QALY }\end{array}$ & + & $5-10 \mathrm{~min}$ & Not stated \\
\hline $\begin{array}{l}\text { Lock et al. } \\
\text { (20) }\end{array}$ & $\begin{array}{l}\text { UK } \\
\text { (England) }\end{array}$ & $\begin{array}{l}\text { EEACT/ } \\
\text { Cost } \\
\text { mini- } \\
\text { mization } \\
\text { analysis }\end{array}$ & $\begin{array}{l}\text { Screening with AUDIT followed by } \\
\text { either: } \\
\text { (1) Treatment as usual } \\
\text { (2) } 5-10 \text { min nurse-led brief } \\
\text { intervention }\end{array}$ & $\begin{array}{l}\text { Intervention costs, } \\
\text { health and social care } \\
\text { resource use and } \\
\text { personal costs at } \\
12 \text { months follow-up }\end{array}$ & $\begin{array}{l}\text { SF-12 score at } \\
12 \text { months } \\
\text { follow-up }\end{array}$ & $\begin{array}{l}\text { No statistically significant } \\
\text { difference in costs or health } \\
\text { outcomes between arms }\end{array}$ & + & $5-10 \mathrm{~min}$ & Nurse \\
\hline $\begin{array}{l}\text { Ludbrook } \\
\text { et al. (7) }\end{array}$ & $\begin{array}{l}\text { UK } \\
\text { (Scotland) }\end{array}$ & CEA & $\begin{array}{l}\text { (1) Do-nothing scenario } \\
\text { (2) Screening using } 7 \text {-day timeline } \\
\text { follow back followed by } 2 \times 15 \text { min } \\
\text { brief interventions each followed } \\
\text { up with a } 5 \text { min telephone call }\end{array}$ & $\begin{array}{l}\text { Intervention costs, } \\
\text { patient time/travel, } \\
\text { health and social care } \\
\text { resource use, motor } \\
\text { vehicle crashes and } \\
\text { legal/criminal costs over } \\
\text { lifetime horizon }\end{array}$ & $\begin{array}{l}\text { Life years gained } \\
\text { over lifetime } \\
\text { horizon }\end{array}$ & SBI dominates vs. do-nothing & - & $40 \mathrm{~min}$ & $\begin{array}{l}\text { GP (nurse } \\
\text { delivers } \\
\text { follow-up } \\
\text { phone calls) }\end{array}$ \\
\hline $\begin{array}{l}\text { Mundt } \\
\text { et al. (21) }\end{array}$ & USA & $\begin{array}{l}\text { EEACT/ } \\
\text { CBA }\end{array}$ & $\begin{array}{l}\text { Screening with health screening } \\
\text { survey and assessment interview } \\
\text { followed by either: } \\
\text { (1) Treatment as usual } \\
\text { (2) } 2 \times 15 \text { min bried interventions } \\
\text { each followed up with a } 5 \text { min } \\
\text { telephone call }\end{array}$ & $\begin{array}{l}\text { Intervention costs, } \\
\text { patient time/travel and } \\
\text { health and social care } \\
\text { resource use over } \\
2 \text { years follow-up }\end{array}$ & $\begin{array}{l}\text { Life years lost } \\
\text { (valued at } \\
\$ 50,000 \text { each) } \\
\text { over } 2 \text { years } \\
\text { follow-up }\end{array}$ & $\begin{array}{l}\text { Non-significant cost savings of } \\
\$ 467 \text { from healthcare perspective } \\
\text { and } \$ 812 \text { from societal } \\
\text { perspective for } \mathrm{Bl} \text { vs. treatment as } \\
\text { usual }\end{array}$ & + & $40 \mathrm{~min}$ & $\begin{array}{l}\text { GP (nurse } \\
\text { delivers } \\
\text { follow-up } \\
\text { phone calls) }\end{array}$ \\
\hline
\end{tabular}


Table 1 | Continued

\begin{tabular}{|c|c|c|c|c|c|c|c|c|c|}
\hline Study & Country & $\begin{array}{l}\text { Study } \\
\text { type }\end{array}$ & Comparators & Costs included & $\begin{array}{l}\text { Health } \\
\text { outcomes } \\
\text { included }\end{array}$ & Results & Quality & $\begin{array}{l}\text { Duration of } \\
\text { intervention }\end{array}$ & $\begin{array}{l}\text { BI delivery } \\
\text { staff }\end{array}$ \\
\hline $\begin{array}{l}\text { Navarro } \\
\text { et al. (24) }\end{array}$ & Australia & CEA & $\begin{array}{l}\text { (1) Current level of SBI provision } \\
\text { (2) Increased levels of screening } \\
\text { and brief intervention or combined } \\
\text { SBI provision }\end{array}$ & $\begin{array}{l}\text { Intervention costs } \\
\text { (including training) }\end{array}$ & $\begin{array}{l}\text { Number of risky } \\
\text { drinkers who } \\
\text { reduce their } \\
\text { alcohol } \\
\text { consumption }\end{array}$ & $\begin{array}{l}\text { Additional cost of between } \\
\$ 174-1041 \text { per risky drinker who } \\
\text { reduces their drinking, depending } \\
\text { on the scenario }\end{array}$ & + & Not stated & GP \\
\hline $\begin{array}{l}\text { Purshouse } \\
\text { et al. (4) }\end{array}$ & $\begin{array}{l}\text { UK } \\
\text { (England) }\end{array}$ & CUA & $\begin{array}{l}\text { (1) Do-nothing scenario } \\
\text { (2) Screening with AUDIT followed } \\
\text { by } 5 \text { min brief intervention }\end{array}$ & $\begin{array}{l}\text { Intervention costs and } \\
\text { health and social care } \\
\text { resource use over } \\
30 \text { years following start } \\
\text { of program }\end{array}$ & $\begin{array}{l}\text { QALYs gained } \\
\text { over } 30 \text { years } \\
\text { follow-up }\end{array}$ & $\begin{array}{l}\text { SBI delivered at next GP } \\
\text { registration dominates do-nothing } \\
\text { scenario. SBI at next GP } \\
\text { consultation has an ICER of } £ 1175 \\
\text { per QALY vs. do-nothing }\end{array}$ & ++ & $5 \mathrm{~min}$ & $\begin{array}{l}\text { Practice } \\
\text { nurse/GP } \\
\text { (both } \\
\text { modeled) }\end{array}$ \\
\hline $\begin{array}{l}\text { Rehm } \\
\text { et al. ( } 27)\end{array}$ & Canada & CBA & $\begin{array}{l}\text { (1) Do-nothing scenario } \\
\text { (2) Screening followed by brief } \\
\text { intervention }\end{array}$ & $\begin{array}{l}\text { Health and social care } \\
\text { resource use costs, } \\
\text { costs of crime and } \\
\text { productivity losses due } \\
\text { to death and disability } \\
\text { per annum. Unclear if } \\
\text { intervention costs are } \\
\text { included }\end{array}$ & $\begin{array}{l}\text { Deaths, years of } \\
\text { life lost and } \\
\text { acute hospital } \\
\text { days averted per } \\
\text { annum }\end{array}$ & $\begin{array}{l}\text { Introduction of BI would avoid } 360 \\
\text { deaths, } 9000 \text { years of life lost, } \\
56,000 \text { acute care hospital days } \\
\text { and would reduce } \\
\text { alcohol-attributable costs by } \\
\$ 602 \text { m per annum vs. do-nothing }\end{array}$ & + & Not stated & Not stated \\
\hline $\begin{array}{l}\text { Saitz et al. } \\
\text { (31) }\end{array}$ & USA & CUA & $\begin{array}{l}\text { (1) Do-nothing scenario } \\
\text { (2) Screening followed by brief } \\
\text { intervention }\end{array}$ & $\begin{array}{l}\text { Intervention costs and } \\
\text { health and social care } \\
\text { resource use over } \\
\text { lifetime horizon }\end{array}$ & $\begin{array}{l}\text { QALYs gained } \\
\text { over a lifetime } \\
\text { horizon }\end{array}$ & SBI dominates vs. do-nothing & - & Not stated & Not stated \\
\hline $\begin{array}{l}\text { Solberg } \\
\text { et al. (25) }\end{array}$ & USA & CUA & $\begin{array}{l}\text { (1) Do-nothing scenario } \\
\text { (2) Annual screening followed by } \\
5 \mathrm{~min} \mathrm{BI}\end{array}$ & $\begin{array}{l}\text { Intervention costs, } \\
\text { patient time/travel and } \\
\text { health and social care } \\
\text { resource use over } \\
\text { lifetime horizon }\end{array}$ & $\begin{array}{l}\text { QALYs gained } \\
\text { over lifetime } \\
\text { horizon }\end{array}$ & $\begin{array}{l}\text { ICER of } \$ 1750 \text { per QALY vs. } \\
\text { do-nothing with healthcare } \\
\text { perspective. SBI dominates with } \\
\text { societal perspective }\end{array}$ & + & $5 \mathrm{~min}$ & GP \\
\hline $\begin{array}{l}\text { Tariq et al. } \\
\text { (26) }\end{array}$ & Netherlands & CUA & $\begin{array}{l}\text { (1) Do-nothing scenario } \\
\text { (2) Screening with AUDIT followed } \\
\text { by } 10-15 \text { min brief intervention }\end{array}$ & $\begin{array}{l}\text { Intervention costs and } \\
\text { health and social care } \\
\text { resource use costs over } \\
\text { a lifetime horizon }\end{array}$ & $\begin{array}{l}\text { QALYs gained } \\
\text { over lifetime } \\
\text { horizon }\end{array}$ & $\begin{array}{l}\text { ICER of } € 5400 \text { per QALY gained } \\
\text { for brief interventions vs. } \\
\text { do-nothing }\end{array}$ & ++ & $30-45 \min$ & GP \\
\hline
\end{tabular}




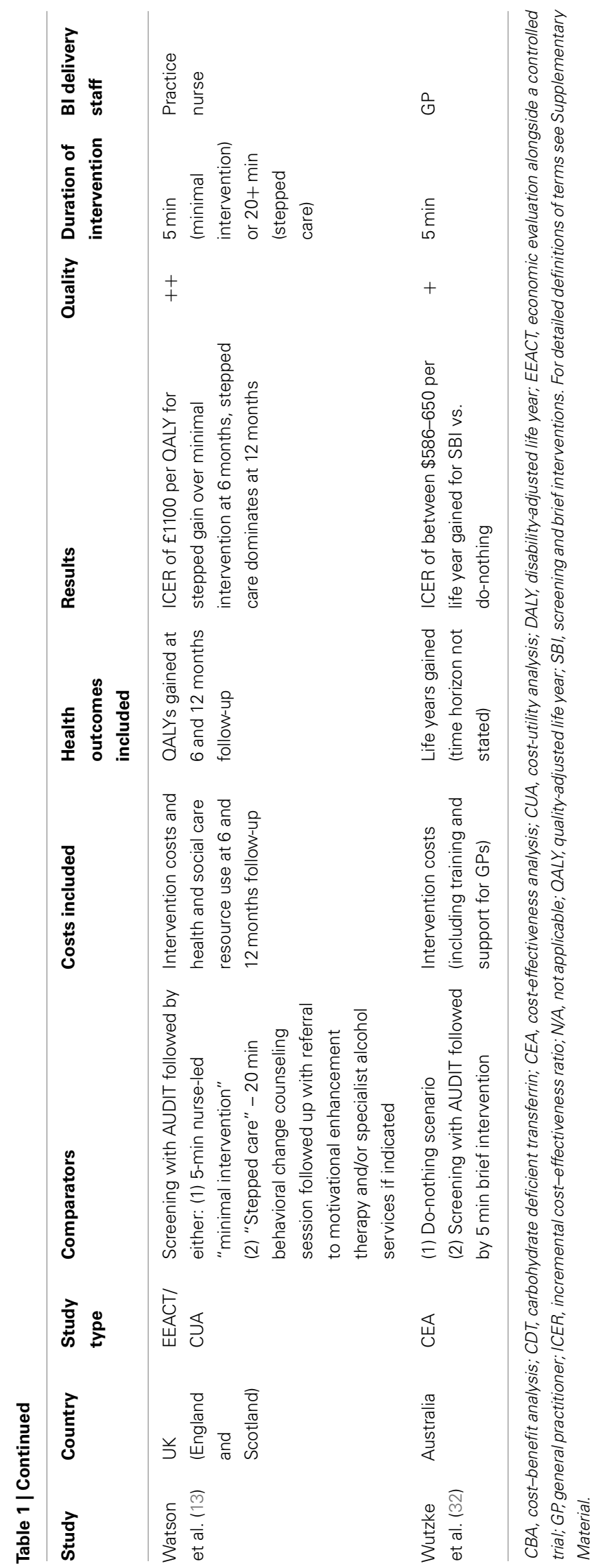

gains, making SBI programs highly likely to be considered costeffective under the relevant national guidelines. The sole exception was Chisholm et al. (30), who presented separate costs and benefits for each of 12 World Health Organization (WHO) sub-regions and found that SBI programs are dominated by current taxation in parts of Africa (region AfrE), although they estimated that they are either cost-effective or cost-saving in the remaining 11 subregions using the WHO's estimated cost-effectiveness thresholds (35). Of the remaining modeling studies, one (27) uses a burden of disease approach to estimate SBIs would be substantially costsaving (Canadian $\$ 602 \mathrm{~m}$ per annum). The remaining studies (24, 29) use intermediate end points (number of risky drinkers averted and change in mean alcohol consumption), which make the results unhelpful for the purpose of informing resource allocation decisions without additional modeling to estimate the impact of these end points on health outcomes. The majority of these modeling studies consider outcomes over a 30 year $(4,28,33)$ or lifetime $(7$, $22,23,25,26,30,31)$ time horizon, ensuring that the long-term impacts are reflected in the results.

Fifteen studies examine the cost-effectiveness of GP-delivered interventions $(4,7,15,16,18,21,22,24-26,28-30,32,33)$, while only five examine nurse-delivered interventions (4, 13-15, 20). Owing to the substantial heterogeneity between studies both in terms of methods and outcomes it is difficult to draw any clear conclusions about the relative cost-effectiveness of using different staff to deliver SBI programs, although the lack of a clear difference between the two options may be of interest to policy makers. Only two studies directly compare both options: Purshouse et al. (4) assume a priori that delivery staff do not impact on the effectiveness of the BI but find that even the use of the more expensive GP-delivered BI option is unlikely to prevent the program from being cost-effective. Meanwhile, Babor et al. (15) conducted a trial with separate nurse-delivered and GP-delivered SBI arms. The authors found no significant difference in effectiveness of the intervention between these arms, while the nurse-delivered option was around $1 / 3$ cheaper, indicating it to be a more cost-effective option.

With regards to the total duration of the intervention (i.e., the total contact time between patient and delivery staff, either faceto-face or over the telephone, aggregated over multiple contacts where appropriate), 12 studies evaluate interventions of $10 \mathrm{~min}$ or less $(4,13-15,20,23,25,28,32,33)$ and 11 consider interventions of over $10 \mathrm{~min}$ (with a maximum duration of $45 \mathrm{~min})(4,7,13$, $14,16,18,21,26,28,29)$. Again the heterogeneity of methods and outcomes makes direct comparison difficult, although there is no clear difference in terms of cost-effectiveness between shorter and longer interventions. Only five studies consider both longer and shorter interventions. Two of these $(13,14)$ report that the longer intervention is cost-effective relative to the shorter one, although this conclusion is difficult to make on the basis of the analysis presented in the studies, particularly given the short follow-up of the trials. The other three studies $(4,28,33)$ assume no difference in effectiveness but find that longer, more expensive interventions are still highly likely to be considered cost-effective compared to no intervention.

In order to further explore the relationship between delivery staff, BI duration, and cost-effectiveness, Figure 2 presents a direct 


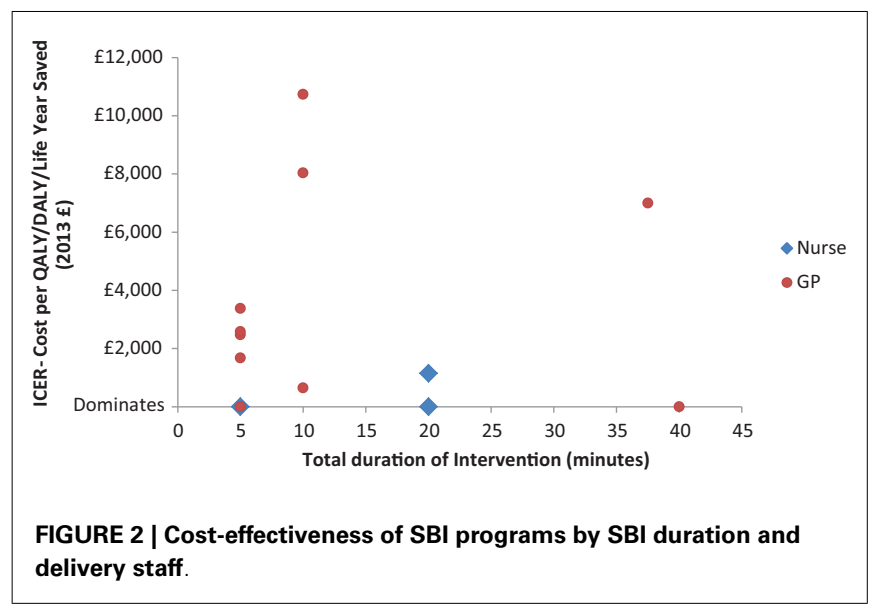

comparison of the cost-effectiveness results converted to $2013 \mathrm{UK}$ $\mathfrak{E}$, for those studies which report delivery staff, intervention duration, and an Incremental Cost-Effectiveness Ratio (ICER) (4, 13, $25,26,28,33,36)$.

\section{DISCUSSION}

This systematic review provides strong evidence that SBIs in a primary care setting are a cost-effective policy option for tackling alcohol-related harms, at least in high-income countries. There is a paucity of evidence for lower- or middle-income countries and that does exist indicates that there may be substantial heterogeneity in both the expected costs and effectiveness of SBI programs depending on the local context in these areas (30).

There is also substantial heterogeneity in study methods, included costs, and reported health outcomes between the included studies, which makes it difficult to determine the implications of this diverse body of evidence for those making resource allocation decisions, although there is an apparent trend for more recent studies to use standardized measures such as QALYs or DALYs, which makes between-study comparison more meaningful. There are also significant differences in the national contexts between studies (for example the existing level of drinking or the current suite of alcohol policies in the country), which must be considered when making international comparisons.

Considering these differences, there is no clear evidence that the choice of delivery staff for SBI programs has a substantial impact on the program's cost-effectiveness. This may be because GP-delivered interventions are more effective but more costly than those delivered by nurses, although this would be at odds with existing literature, which suggests that the use of less costly nursing staff to conduct tasks that would otherwise be the responsibility of GPs is unlikely to impact negatively on the quality of care received by patients $(37,38)$. Figure 2 also suggests that nurses may be a more cost-effective option, although heterogeneity in settings and methods between the included studies mean that the graph should be interpreted with caution.

It is also important to note that policy makers will need to consider the total budget impact of any policy options in addition to the potential cost-effectiveness, an issue highlighted in several of the included studies $(28,33,39)$. This may suggest that nurse-led SBI programs, which are likely to be less costly overall, may be more appealing option, although consideration must be given to the existing primary care systems in each country. For example, in countries such as the UK or the Netherlands where practice nurses already undertake many primary care services such as vaccinations or health checks, nurse-led SBIs may be a more practical option than in other countries where care is currently delivered exclusively by the GP.

There is also no clear evidence that the duration of intervention delivered has a substantial impact on cost-effectiveness. Again this may indicate that longer interventions are more effective but more expensive, although studies on the effectiveness evidence have not found a consistent relationship between amount of patient contact and effectiveness $(5,40)$. While the studies by Watson and Drummond provide limited evidence that longer interventions may be more cost-effective in the short-term in the UK context, it is not clear that this translates to the longer term, or to other countries $(13,14)$.

In addition to the substantial heterogeneity between studies already mentioned, there are a number of limitations to this systematic review. Only studies published in the English language were included, something which may be at least partly responsible for the lack of included studies from the developing world. Some of the included studies are also of low methodological quality which makes it difficult to evaluate the robustness of their conclusions. Finally, there are two key issues, which no study of SBI effectiveness can escape. The first is that the estimates of effectiveness, which underpin the cost-effectiveness estimates examined here may be exaggerated by the impact of regression to the mean, caused by drinkers changing their consumption over time for reasons unrelated to the receipt of a brief intervention (e.g., public holidays or seasonal variation) (41). The second, countervailing issue is that of an intervention or Hawthorne effect, whereby the act of being enrolled into a trial acts as an intervention in itself, something which may at least partly explain why many SBI effectiveness studies observe a reduction in alcohol consumption over time in the control groups (42).

Limitations in the evidence base mean that this review is unable to address a number of other issues that may be of interest to policy makers such as the cost-effectiveness of SBI programs targeting specific groups within the general population. Further research to examine the differential effectiveness of, and the likely coverage by, SBI programs in these subgroups is important to allow this area to be explored further. The other key priority for further research to inform decision makers concerns the uptake among primary care providers of SBI programs. Difficulties in persuading GPs and nurses to fully deliver SBI programs could have a substantial impact on the effectiveness and cost-effectiveness of these programs. A recent international trial conducted as part of the optimizing delivery of healthcare interventions (ODHIN) project will go some way to addressing this challenge by examining the effectiveness and cost-effectiveness of different strategies at increasing SBI delivery rates in primary care (43).

In conclusion, while there are significant differences between the studies included in this review, the overwhelming conclusion is 
that SBIs in primary care are a cost-effective option, at least in highincome countries. There is no clear evidence that the duration of the intervention, or the type of staff used to deliver it, changes this conclusion. Policy makers should, however, be mindful of the differing budget implications that alternative implementation options may present.

\section{AUTHOR CONTRIBUTIONS}

Louise Preston undertook the literature searches. Colin Angus conducted the review, with assistance from Jessica Li, and drafted the article. Robin Purshouse and Nicholas Latimer provided guidance and expertise. All authors read and approved the final manuscript.

\section{ACKNOWLEDGMENTS}

The research leading to these results or outcomes has received funding from the European Union's Seventh Framework Program for research, technological development and demonstration under grant agreement no. 259268 - optimizing delivery of health care intervention (ODHIN). Participant organizations in ODHIN can be seen at www.odhinproject.eu/partners.html. The views expressed here reflect only the authors' and the European Union is not liable for any use that may be made of the information contained therein.

\section{SUPPLEMENTARY MATERIAL}

The Supplementary Material for this article can be found online at http://www.frontiersin.org/Journal/10.3389/fpsyt.2014.00114/ abstract

\section{REFERENCES}

1. Lim SS, Vos T, Flaxman AD, Danaei G, Shibuya K, Adair-Rohani H, et al. A comparative risk assessment of burden of disease and injury attributable to 67 risk factors and risk factor clusters in 21 regions, 1990-2010: a systematic analysis for the Global Burden of Disease Study 2010. Lancet (2012) 380:2224-60. doi:10.1016/S0140-6736(12)61766-8

2. Anderson P, Baumberg B. Alcohol in Europe. London (2006). Available from: http://leonardo3.dse.univr.it/addiction/documents/External/alcoholineu.pdf

3. Cherpitel CJ. Drinking patterns and problems among primary care patients: a comparison with the general population. Alcohol Alcohol (1991) 26:627-33.

4. Purshouse R, Brennan A, Rafia R, Latimer NR, Archer RJ, Angus CR, et al. Modelling the cost-effectiveness of alcohol screening and brief interventions in primary care in England. Alcohol Alcohol (2013) 48:180-8. doi:10.1093/alcalc/ags103

5. O'Donnell A, Anderson P, Newbury-Birch D, Schulte B, Schmidt C, Reimer J, et al. The impact of brief alcohol interventions in primary healthcare: a systematic review of reviews. Alcohol Alcohol (2013) 49:66-78. doi:10.1093/alcalc/ agt170

6. World Health Organization. Global Strategy to Reduce the Harmful Use of Alcohol. Available from: http://www.who.int/substance_abuse/alcstratenglishfinal. pdf?ua $=1$

7. Ludbrook A, Godfrey C, Wyness L, Parrott S, Haw S, Napper M, et al. Effective and Cost-Effective Measures to Reduce Alcohol Misuse in Scotland: A Literature Review. Aberdeen: Health Economics Research Unit (2001). Available from: http://www.alcoholinformation.isdscotland.org/alcohol_misuse/files/measure reduce_full.pdf

8. Latimer N, Guillaume L, Goyder E. Prevention and Early Identification of Alcohol Use Disorders in Adults and Young People. Screening and Brief Interventions: Cost Effectiveness Review. Sheffield (2008). Available from: http://www.sheffield.ac. uk/polopoly_fs/1.43294!/file/Alcohol-2_3.pdf

9. Elzerbi C, Donoghue K, Drummond C. Report on the European Public Health Impact and Cost Effectiveness of Early Diagnosis and Treatment of Alcohol Use Disorders (2013). Available from: http://amphoraproject.net/w2box/data/ Deliverables/AMPHORA_WP6_D3.3.pdf
10. Wojnar M. Optimizing Delivery of Health Care Interventions (ODHIN): Survey of Attitudes and Managing Alcohol Problems in General Practice in Europe Final Report. Warsaw (2014). Available from: http://www.odhinproject.eu/ resources/documents/doc_download/52-deliverable-4-1-survey-of-attitudesand-managing-alcohol-problems-in-general-practice-in-europe.html

11. Drummond MF, Jefferson TO. Guidelines for authors and peer reviewers of economic submissions to the BMJ. The BMJ Economic Evaluation Working Party. BMJ (1996) 313:275-83. doi:10.1136/bmj.313.7052.275

12. The Cochrane Collaboration. Cochrane Handbook for Systematic Reviews of Interventions. 5th ed. Higgins JP, Green S, editors (2011). Available from: www.cochrane-handbook.org

13. Watson J, Crosby H, Dale V, Tober G, Wu Q, Lang J, et al. AESOPS: a randomised controlled trial of the clinical effectiveness and cost-effectiveness of opportunistic screening and stepped care interventions for older hazardous alcohol users in primary care. Health Technol Assess (2013) 17:1-158. doi:10.3310/hta17250

14. Drummond C, Coulton S, James D, Godfrey C, Parrott S, Baxter J, et al. Effectiveness and cost-effectiveness of a stepped care intervention for alcohol use disorders in primary care: pilot study. Br J Psychiatry (2009) 195:448-56. doi:10.1192/bjp.bp.108.056697

15. Babor TF, Higgins-Biddle JC, Dauser D, Burleson JA, Zarkin GA, Bray J. Brief interventions for at-risk drinking: patient outcomes and cost-effectiveness in managed care organizations. Alcohol Alcohol (2006) 41:624-31. doi:10.1093/ alcalc/agl078

16. Dillie KS, Mundt M, French MT, Fleming MF. Cost-benefit analysis of a new alcohol biomarker, carbohydrate deficient transferrin, in a chronic illness primary care sample. Alcohol Clin Exp Res (2005) 29:2008-14. doi:10.1097/01.alc. 0000187606.54854.db

17. Fleming MF, Mundt MP, French MT, Manwell LB, Stauffacher EA, Barry KL. Benefit-cost analysis of brief physician advice with problem drinkers in primary care settings. Med Care (2000) 38:7-18. doi:10.1097/00005650-20000100000003

18. Fleming M, Mundt M, French M, Manwell L, Stauffacher E, Barry K. Brief physician advice for problem drinkers: long-term efficacy and benefit-cost analysis. Alcohol Clin Exp Res (2002) 26:36-43. doi:10.1111/j.1530-0277.2002.tb02429.x

19. Freeborn DK, Polen MR, Hollis JF, Senft RA. Screening and brief intervention for hazardous drinking in an HMO: effects on medical care utilization. J Behav Health Serv Res (2000) 27:446-53. doi:10.1007/BF02287826

20. Lock CA, Kaner EF, Heather N, Doughty J, Crawshaw A, McNamee P, et al. Effectiveness of nurse-led brief alcohol intervention: a cluster randomized controlled trial. J Adv Nurs (2006) 54:426-39. doi:10.1111/j.1365-2648.2006.03836.x

21. Mundt MP, French MT, Roebuck MC, Manwell LB, Barry KL. Brief physician advice for problem drinking among older adults: an economic analysis of costs and benefits. J Stud Alcohol (2005) 66:389-94.

22. Cobiac L, Vos T, Doran C, Wallace A. Cost-effectiveness of interventions to prevent alcohol-related disease and injury in Australia. Addiction (2009) 104:1646-55. doi:10.1111/j.1360-0443.2009.02708.x

23. Kapoor A, Kraemer KL, Smith KJ, Roberts MS, Saitz R. Cost-effectiveness of screening for unhealthy alcohol use with \% carbohydrate deficient transferrin: results from a literature-based decision analytic computer model. Alcohol Clin Exp Res (2009) 33:1440-9. doi:10.1111/j.1530-0277.2009.00974.x

24. Navarro HJ, Shakeshaft A, Doran CM, Petrie DJ. The potential cost-effectiveness of general practitioner delivered brief intervention for alcohol misuse: evidence from rural Australia. Addict Behav (2011) 36:1191-8. doi:10.1016/j.addbeh. 2011.07.023

25. Solberg LI, Maciosek MV, Edwards NM. Primary care intervention to reduce alcohol misuse ranking its health impact and cost effectiveness. Am J Prev Med (2008) 34:143-52. doi:10.1016/j.amepre.2007.09.035

26. Tariq L, Van Den Berg M, Hoogenveen RT, Van Baal PH. Cost-effectiveness of an opportunistic screening programme and brief intervention for excessive alcohol use in primary care. PLoS One (2009) 4:8. doi:10.1371/journal.pone.0005696

27. Rehm J, Gnam WH, Popova S, Patra J, Sarnocinska-Hart A. Avoidable Cost of Alcohol Abuse in Canada 2002. Toronto, ON (2008). Available from: http://www.researchgate.net/publication/49676020_Avoidable_cost_of_ alcohol_abuse_in_Canada/file/d912f50b9440fa6bef.pdf

28. Angus C, Scafato E, Ghirini S, Torbica A, Ferre F, Struzzo P, et al. Costeffectiveness of a programme of screening and brief interventions for alcohol in primary care in Italy. BMC Fam Pract (2014) 15:26. doi:10.1186/1471-229615-26 
29. Freemantle N, Gill P, Godfrey C, Long A, Richards C, Sheldon TA, et al. Brief interventions and alcohol use. Qual Health Care (1993) 2:267-73. doi:10.1136/ qshc. 2.4 .267

30. Chisholm D, Rehm J, van Ommeren M, Monteiro M. Reducing the global burden of hazardous alcohol use: a comparative cost-effectiveness analysis. J Stud Alcohol (2004) 65:782-93.

31. Saitz R, Svikis D, D’Onofrio G, Kraemer KL, Perl H. Challenges applying alcohol brief intervention in diverse practice settings: populations, outcomes, and costs. Alcohol Clin Exp Res (2006) 30:332-8. doi:10.1111/j.1530-0277.2006.00038.x

32. Wutzke SE, Shiell A, Gomel MK, Conigrave KM. Cost effectiveness of brief interventions for reducing alcohol consumption. Soc Sci Med (2001) 52:863-70. doi:10.1016/S0277-9536(00)00189-1

33. Angus C, Scafato E, Ghirini S, Torbica A, Ferre F, Struzzo P, et al. Optimizing Delivery of Health Care Interventions (ODHIN): Cost-Effectiveness Model Report. Sheffield (2013). Available from: http://www.odhinproject.eu/ resources/documents/doc_download/66-deliverable-3-1-cost-effectivenessmodel-report.html

34. Holmes J, Meier PS, Booth A, Guo Y, Brennan A. The temporal relationship between per capita alcohol consumption and harm: a systematic review of time lag specifications in aggregate time series analyses. Drug Alcohol Depend (2012) 123:7-14. doi:10.1016/j.drugalcdep.2011.12.005

35. WHO. Table: Threshold Values for Intervention Cost-Effectiveness by Region (2014). Available from: http://www.who.int/choice/costs/CER_levels/en/

36. Ludbrook A. Effective and Cost - Effective Measures to Reduce Alcohol Misuse in Scotland: An Update to the Literature Review. Scottish Government (2004). Available from: http://www.scotland.gov.uk/Publications/2005/ 01/20542/50228

37. Laurant M, Reeves D, Hermens R, Braspenning J, Grol R, Sibbald B. Substitution of doctors by nurses in primary care. Cochrane Database Syst Rev (2005) (2):CD001271. doi:10.1002/14651858.CD001271.pub2

38. Laurant M, Harmsen M, Wollersheim H, Grol R, Faber M, Sibbald B. The impact of nonphysician clinicians: do they improve the quality and costeffectiveness of health care services? Med Care Res Rev (2009) 66:36S-89S. doi:10.1177/1077558709346277

39. Meier PS, Meng Y, Holmes J, Baumberg B, Purshouse R, Hill-McManus D, et al. Adjusting for unrecorded consumption in survey and per capita sales data: quantification of impact on gender- and age-specific alcohol-attributable fractions for oral and pharyngeal cancers in Great Britain. Alcohol Alcohol (2013) 48:241-9. doi:10.1093/alcalc/agt001

40. Kaner EF, Beyer F, Dickinson HO, Pienaar E, Campbell F, Schlesinger C, et al. Effectiveness of brief alcohol interventions in primary care populations. Cochrane Database Syst Rev (2007) 4:CD004148. doi:10.1002/14651858. CD004148.pub3

41. Jenkins RJ, McAlaney J, McCambridge J. Change over time in alcohol consumption in control groups in brief intervention studies: systematic review and meta-regression study. Drug Alcohol Depend (2009) 100:107-14. doi:10.1016/j. drugalcdep.2008.09.016

42. McCambridge J, Kypri K. Can simply answering research questions change behaviour? Systematic review and meta analyses of brief alcohol intervention trials. PLoS One (2011) 6:e23748. doi:10.1371/journal.pone.0023748

43. Keurhorst MN, Anderson P, Spak F, Bendtsen P, Segura L, Colom J, et al. Implementing training and support, financial reimbursement, and referral to an internet-based brief advice program to improve the early identification of hazardous and harmful alcohol consumption in primary care (ODHIN): study protocol for a cluster rando. Implement Sci (2013) 8:11. doi:10.1186/17485908-8-11

Conflict of Interest Statement: The authors declare that the research was conducted in the absence of any commercial or financial relationships that could be construed as a potential conflict of interest.

Received: 30 June 2014; accepted: 12 August 2014; published online: 01 September 2014. Citation: Angus C, Latimer N, Preston L, Li J and Purshouse R (2014) What are the implications for policy makers? A systematic review of the cost-effectiveness of screening and brief interventions for alcohol misuse in primary care. Front. Psychiatry 5:114. doi: 10.3389/fpsyt.2014.00114

This article was submitted to Addictive Disorders and Behavioral Dyscontrol, a section of the journal Frontiers in Psychiatry.

Copyright (C) 2014 Angus, Latimer, Preston, Li and Purshouse. This is an open-access article distributed under the terms of the Creative Commons Attribution License (CC $B Y)$. The use, distribution or reproduction in other forums is permitted, provided the original author(s) or licensor are credited and that the original publication in this journal is cited, in accordance with accepted academic practice. No use, distribution or reproduction is permitted which does not comply with these terms. 